\title{
Regenerative Corneal Prosthesis as an Alternative to Human Donor Cornea for Transplantation to Treat Blindness
}

\section{Shivam Bajoria*}

Teerthanker Mahaveer University, Moradabad, India

*Corresponding Author: Shivam Bajoria, Teerthanker Mahaveer University, Moradabad, India.
Received: August 31, 2021

Published: November 09, 2021

(C) All rights are reserved by Shivam Bajoria.

\begin{abstract}
An alternative to human donor cornea is needed as the availability of limited good quality tissue, with this condition potentially worsening in many countries as the population is progressively ageing. Corneal equivalent develop by numerous attempts as an alternative to human corneas donated as well as prosthesis. In this review the efforts in bioengineering implants has been focus that are described include biomaterials that allow for partial regeneration of corneal tissue, self-assembled cornea constructs and cell free. Cornea implants that promoted regeneration when evaluated in clinical trials.
\end{abstract}

Keywords: Cornea; Biomaterials; Prostheses; Regeneration; Self Assembly; Implants; Clinical Studies

\section{Introduction}

The human cornea is the optically clear window of an eye and the main refractive component that focuses light to the Retina allowing vision.

Thus, the transparency of the cornea optically is critical for vision optimally, injury or disease that causes loss of transparency irreversibly lead to vision loss and eventually blindness. It is estimated globally that 4.9 million individuals have bilateral corneal blindness while unilateral corneal blind are estimated by 23 million.

Human donor cornea with corneal transplantation is the only extensively accepted to restore eyesight. Whereas, factually human cornea is one of the most transplanted tissues worldwide, with over 53000 corneal grafts performed in a particular country in 2013.
However, its increasingly difficult for eye banks worldwide to meet the growing demand for Eye Banks worldwide donated Cornea, which is in part due to ageing population. The number of Corneal Transplantation performed in a particular country has increased by $50.9 \%$ since 2005 , accordingly to Eye faster than the number of potential donors. Thus, shortage of donor tissue has become a common concern for most countries.

Artificial corneas such as the Alpha Cor Keratoprosthesis are made from Traditional plastics but the most cornea do not completely get Biointegrated. The Boston Keratoprosthesis and Osteoporosis Odontokeratoprosthesis and Osteo-Odontokeratoprosthesis (OOKP) have a plastic optic but patients remaining cornea. It has been reported that these two biological interfaced devices are among the most successful prostheses, the minimal function and Antibiotic therapy is require lifetime along with Immune sup- 
pression immune suppression. The risk of other sight threatening conditions surgical procedure is required such as alternative of eye pressure is placement to a shunt.

Currently, when a Replacement occur in Cornea by Transplantation. The surgical removal of pathological tissue which is removed and replaced by a donated human eye bank cornea that is glued or sutured in place. The success rate overall is high in many of the countries, with first 2 years the rejection rate is only $10-15 \%$ postoperative. What is not commonly reported is that Transplantation results are still sub-optimal. In some cases the immune rejection is problematic and it can lead to graft failure. Graft tissue appositioning can result in Astigmatism if the fit is not precise. As the Graft Integration is slow for up to 1 year the sutures are often left up and these can cause infection and become loose.

With severe pathologies for high risk patients with inflammation, complications and failure rates have been as high as $49 \%$ multiple surgeries are often needed and the prospect of complete vision loss is being face by the patients as each subsequent transplant falls.

\section{Bioengineered substitutes as cornea replacement}

While a plateau for optimising outcomes have practically reached by Corneal surgeons, following traditional corneal transplantation using human donor corneas. While range of new opportunities is being potentially offer Biomaterials technology as new design and materials are possible. Other advantages that potentially bioengineered corneal substitutes might have over donated Human cornea include the following.

Production of large scale of biosynthetic implants offers a potentially person solution to the severe shortage of human donor corneas worldwide:

1. The need for expensive screening for diseases transmitted by disease transmitted by donors and for tissue quality assessment has been eliminated by good manufacturing techniques.

2. The risk of graft failure and eliminating the chronic post operative immunosuppression.

3. The 3-D shape of the corneal substitute get tailored of the same shape as contact lenses and Intra ocular aiming to be
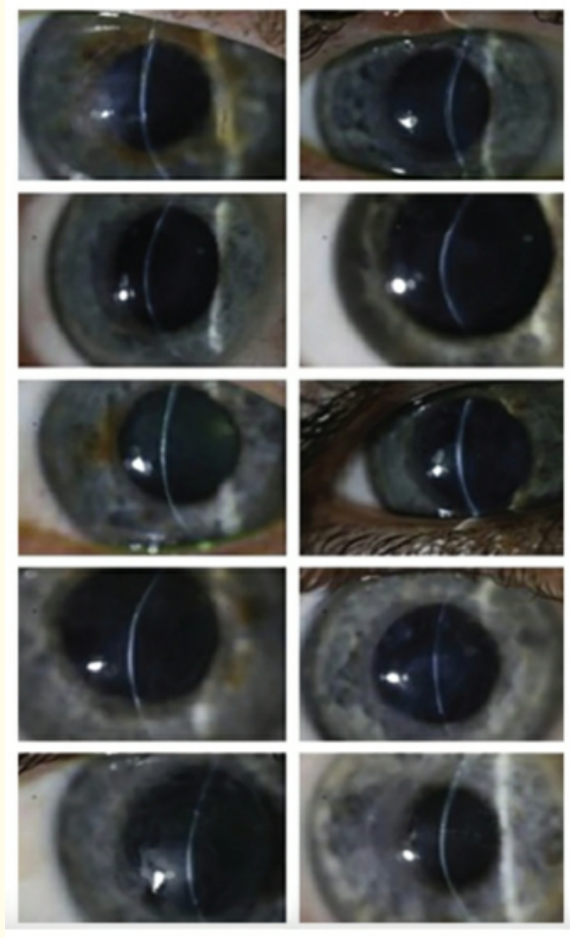

Figure 1: Slit lamp biomicroscopy images of the corneas of all 10 patients at 4 years after grafting with a biosynthetic implant.

more successful post operative refractive results, as it determined many of eye total refractive power and hence visual acuity.

4. The materials biomechanical properties can be adapted to the clinical need e.g. they can be made softer elastic or stronger and firmer or the transportation of fluid and solids can be allow and it therefore do not affect IOP measurements.

5. The design biomaterials are so to interact with cells microorganisms or molecules by the release of bioactives. Various Keratoprosthesis and bioengineered corneal substitutes that have been developed as replacements for pathological corneas and/or to promote regeneration have been received by many others. The contributions of Canadian researchers to the development of technologies for alternative to donor transplantation are only being review in this article. Among 
bioengineered corneal implants reached clinical evaluation in a clinical trial.

Keratoprosthesis with regenerative capability

The focussing on replacement of minimal function to allow vision has been mentioned in traditional Keratoprosthesis.

Sherdown and co0workers have focussed on Keratoprosthesis with allow partial regeneration, by modifying biomaterials with cell adhesion peptides and growth factors. The list of examples of biomaterials developed for use as Keratoprosthesis.

In Aucoin et Al poly (dimethyl siloxane) (PDMS) surfaces got modified by combinations of covalent attachment of cell adhesive peptides that derived from Laminin and fibronectin. YIGSR studies commonly gets included by peptides studies and it's synergistic peptide PDGSR from Laminin and fibronectin derived RGDS and PHSRN. The exponential adhesion statistical analysis results suggested the YIGSR, RGDS and PHSRN used concentrations, as well as YIGSR and PGDSR synergistic affect, had effects significantly on cell attachment and proliferation. The superior adhesion get resulted with multiple peptides and by the use of surface modifications and proliferation of corneal epithelial cells over surfaces modified with single peptides only. The combinations of synergistic peptides potentially results in enhanced cell surface interactions shown by the results.

In Liu and Sherdown the hydrogels high water permeability modelled with poly ( $\mathrm{N}$ - isopropyl acrylbromide), gets combine with high permeable oxygen of poly dimethyl siloxane (silicone) in an interpreted network (IPN). Mechanical strength is possess by resulting IPN that superior to each individual of polymer. The interfacial agents gets incorporated results in better transparency than individualised polymer and an adequate corneal epithelial cell compatibility is demonstrated by these materials. While limited testing has been provided under in Vivo conditions with these materials.

Self0assembled corneal constructs developed a technique fundamentally for developing self assembly of tissues by inducing target cells cultured to synthesise it's own extracellular matrix (ECM). For constructed cornea the use of Ascorbic acid increases to stimulate the production of ECM and collagen by cultured stromal cells. Sheets of ECM and collagen by cultured stromal cells. Sheets

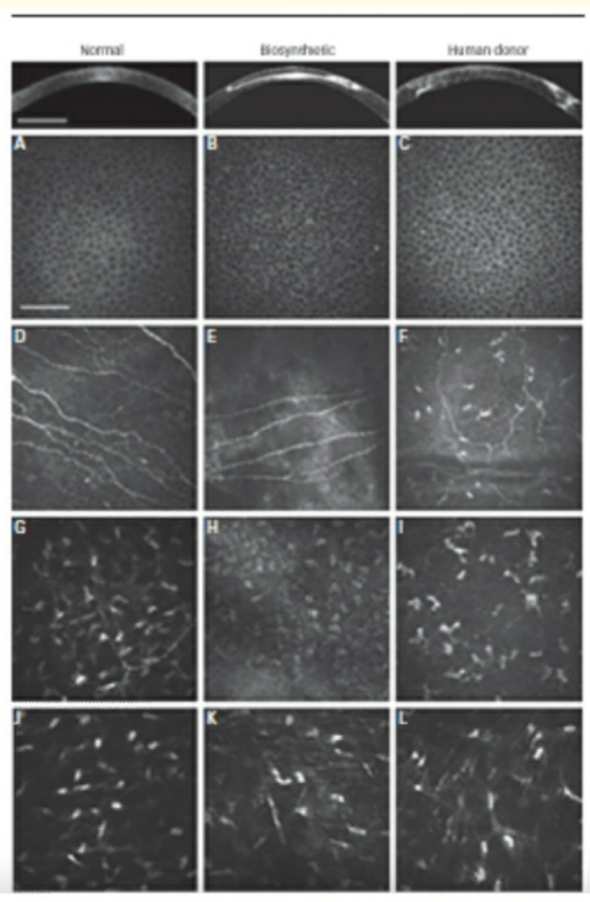

Figure 2: Corneal features in a healthy, unoperated subject, alongside those of operated patients, at 24 months after implantation of a biosynthetic cornea or a human donor cornea.

of ECM secretion can get along to form a thick stack together and a top seeded epithelium. Sheets of a particular morphology and function reproduce some main features of human cornea.

Till date constructs of self assembled cornea have developed that reproduces multiple layers of human cornea. A tissue engineered replacement of stroma was tested in cats recently and after 4 months post-operative each grafts get stable and clear. The implants were reinnervated showing comparable touch sensitivity to pre-operation levels. A lamellar structure evaluated histologically similar to that of a healthy cornea.

First demonstration of regeneration of the Human Cornea promoted by a cell-free implant. 


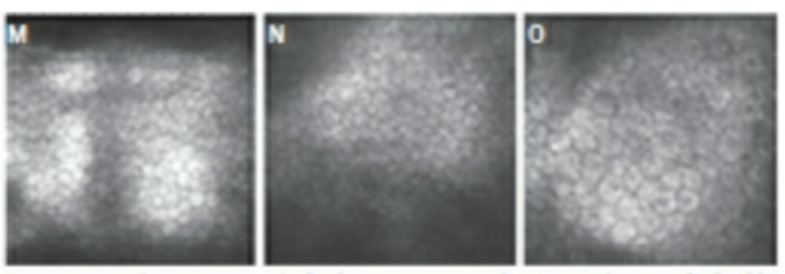

Top row Anterior segnent opocal crherence imography (ASOCD) imges of a heselhy

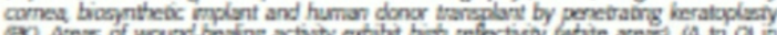

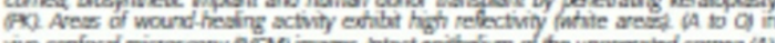
vivo confocal micrascapy $0 \mathrm{OCN}$ imajes intact epithelum of the uncperated cornea iN. regunerated comesl epathedial celt on the implart arface (i) and regenerated equthefium of the penetrating gat in. Repmerated neves if $x$ the sibbesd eqithelum in an mplaned cornes were poralk and morphologicaly amis to the normal comea (D) wheress regenerated subbusal neves were sto obrened in a cornea transplanted with

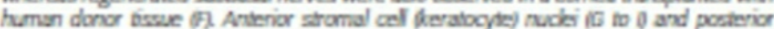
beranocyess $U$ to $U$ were presert with vorying density in al comess the endotolum $M$

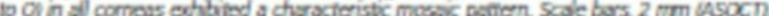
$100 \mathrm{~mm} \mathrm{MCN}$. Reproduced with permission from fogerholm ef al, $2010=$

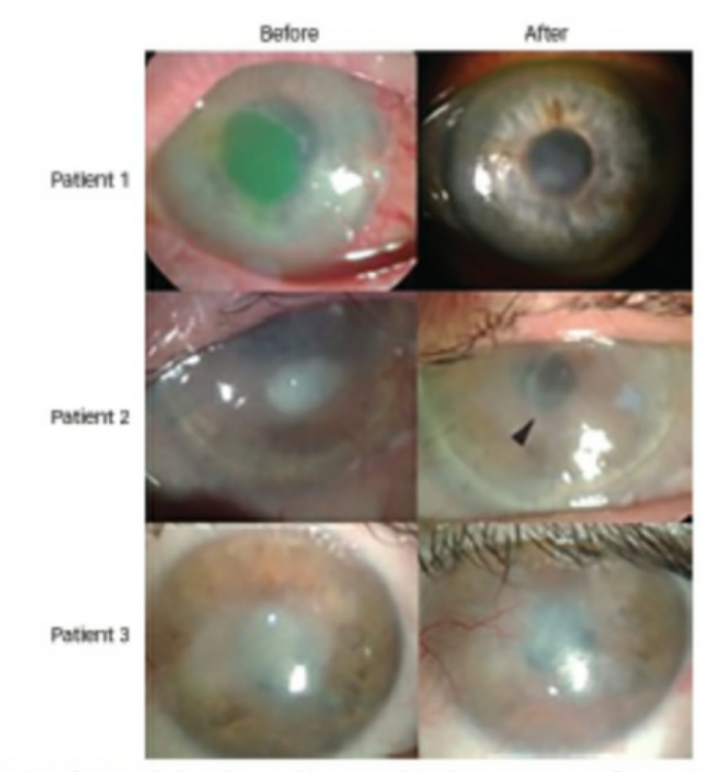

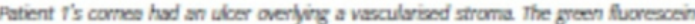
stiving dolineates the lare sres of eroded epithelum. At 12 months post coperabont

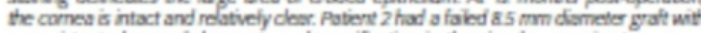
a persistent uloer and derse strond apocification in the viad zore pricr to surpm.

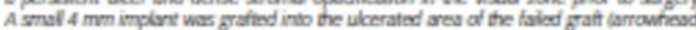

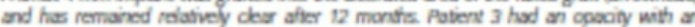

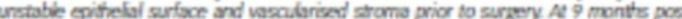

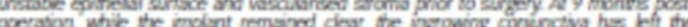

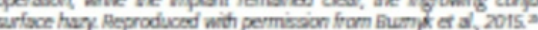

Figure 3: Corneas of all three patients before and after implantation with tectonic grafts of RHCIII-MPC.

A recently published 4 year follow up results of a clinical study Phase-I where transparent analogues biosynthetically analogues of human corneal stromal extracellular matrices, comprises carbodi- imide-crosslinked recombinant human collagen type III (RHC III), were implanted in the first 10 human patients. It has been showed that the implants were stably integrated without immunosuppression. Unlikely, the Donor Corneas doesn't attract antigen presenting dendritic cells. Further the corneal ECM get mimic it promotes the growth patients corneal epithelial cells, Nerves and stromal Keratoconus to regenerate a Neo-Cornea.

It is an important step in corneal transplantation as it get demonstrated for very first time that regeneration may occur in the Human Cornea and it get stimulated by Biomaterials as a donor human allograft alternative. It provide hope to patient who doesn't get transplanted due to worldwide shortage of donor tissues.

Further developing implants include the use of Riboflavin and UV-A light to crosslink potentially or implant welding into the cornea of hosts. In a study conducted by Wand., et al. RHC III corneal implants got successfully, crosslinked into excise porcine and rabbit eyes. While optimising further required to address shrinkage that noted after cross-country linking. Nevertheless, in the future. A reduce suture related complications such as haze formation and surface irregularity after grafting of bioengineered collagen-based implants by this technique.

Bio-engineered cornea implants for high risk patients

Firstly, substitutes RHC III were implanted in Non-inflamed corneas to ensure that Next generation of implants will resist the inflamed eyes resist which includes collagenases, dryness and Neovascularisation that typically lead to ulceration, melting and opacification of the graft the RHC III implants were reinforced. A second network of 2- methacrylate Thule phosphoryl holiness (MPC) crosslinked with polyethylene (glycol) diacrylate (PEGDA) was added to form an network RHC III MPC (Interpreting) implants but optimising for clinical evaluation.

RHC III implants optimised generation were tested in a clinical study.

Three of the patients with ulceration and erosions of corneal surface and whose rejection were at high risk of rejection of human donor corneas were implanted with RHC III-MPC as tectonic grafts. All of the three patients showed symptomatic relief of pain, irritation and photophobia. The implants were still stably integrated at 
9 to 12 months in the patients. An improvement in vision is shown by two our of three patients [1-5].

\section{Conclusion}

The field of cornea regeneration is one that is active and many groups are working on the development of new technologies. It has been shown how several technologies developed have now shown clinical evaluation by ongoing research.

\section{Bibliography}

1. Olivia MS., et al. "Turning the Tide of Corneal Blindness". Indian Journal of Ophthalmology 60.5 (2012;): 423-427.

2. Lohiyo S., et al. "Eye banking and corneal transplantation in tertiary care hospital located in rural areas". IOSR Journal of Pharmacy (IOSRPHR) 4.4 (2014):11-16.

3. Eye bank association, 2013 Eye banking statistical report (2014).

4. Eye bank Association 2009 eye banking statistical report (2010).

5. Mark Just., et al. "Obstacles to donor eye procurement and their solutions at the university of Laws". Cornea 14.3 (1995): 249-252.

Volume 4 Issue 12 December 2021

(C) All rights are reserved by Shivam Bajoria. 